\title{
Influence of geolocation and ethnicity on the phenotypic expression of primary Sjögren's syndrome at diagnosis in 8310 patients: a cross-sectional study from the Sjögren Big Data Project
}

Pilar Brito-Zerón ${ }^{1,2}$, Nihan Acar-Denizli ${ }^{3}$, Margit Zeher ${ }^{4}$, Astrid Rasmussen ${ }^{5}$, Raphaele Seror ${ }^{6}$, Elke Theander ${ }^{7}$, Xiaomei Li ${ }^{8}$, Chiara Baldini ${ }^{9}$, Jacques-Eric Gottenberg ${ }^{10}$, Debashish Danda ${ }^{11}$, Luca Quartuccio ${ }^{12}$, Roberta Priori ${ }^{13}$, Gabriela Hernandez-Molina ${ }^{14}$, Aike A. Kruize ${ }^{15}$, Valeria Valim ${ }^{16}$, Marika Kvarnstrom ${ }^{17}$, Damien Sene ${ }^{18}$, Roberto Gerli $^{19}$, Sonja Praprotnik ${ }^{20}$, David Isenberg ${ }^{21}$, Roser Solans ${ }^{22}$, Maureen Rischmueller ${ }^{23}$, Seung-Ki Kwok ${ }^{24}$, Gunnel Nordmark ${ }^{25}$, Yasunori Suzuki ${ }^{26}$, Roberto Giacomelli27, Valerie Devauchelle-Pensec ${ }^{28}$, Michele Bombardieri29, Benedikt Hofauer ${ }^{30}$, Hendrika Bootsma ${ }^{31}$, Johan G. Brun ${ }^{32}$, Guadalupe Fraile ${ }^{33}$, Steven E. Carsons ${ }^{34}$, Tamer A Gheita $^{35}$, Jacques Morel ${ }^{36}$, Cristina Vollenveider ${ }^{37}$, Fabiola Atzeni ${ }^{38}$, Soledad Retamozo ${ }^{39}$, Ildiko Fanny Horvath ${ }^{4}$, Kathy Sivils ${ }^{5}$, Thomas Mandl7, Pulukool Sandhya ${ }^{11}$, Salvatore de Vita ${ }^{12}$, Jorge Sanchez-Guerrero ${ }^{14}$, Eefje van der Heijden $^{15}$, Virginia Fernandes Moça Trevisani ${ }^{40}$, Marie Wahren-Herlenius ${ }^{17}$, Xavier Mariette ${ }^{6}$, Manuel RamosCasals ${ }^{2,41}$, on behalf of the EULAR-SS Task Force Big Data Consortium*

\begin{abstract}
${ }^{1}$ Autoimmune Diseases Unit, Department of Medicine, Hospital CIMA- Sanitas, Barcelona, Spain.
${ }^{2}$ Sjögren Syndrome Research Group (AGAUR), Laboratory of Autoimmune Diseases Josep Font, IDIBAPS-CELLEX, Department of Autoimmune Diseases, ICMiD, University of Barcelona, Hospital Clínic, Barcelona, Spain.

${ }^{3}$ Department of Statistics, Faculty of Science and Letters, Mimar Sinan Fine Arts University, Istanbul, Turkey. ${ }^{4}$ Division of Clinical Immunology, Faculty of Medicine, University of Debrecen, Debrecen, Hungary.

${ }^{5}$ Arthritis and Clinical Immunology Research Program, Oklahoma Medical Research Foundation, Oklahoma City, OK, USA.
\end{abstract}

${ }^{6}$ Center for Immunology of Viral Infections and Autoimmune Diseases, Assistance Publique - Hôpitaux de Paris, Hôpitaux Universitaires Paris-Sud, Le Kremlin-Bicêtre, Université Paris Sud, INSERM, Paris, France.

${ }^{7}$ Department of Rheumatology, Malmö University Hospital, Lund University, Lund, Sweden.

${ }^{8}$ Department of Rheumatology and Immunology, Anhui Provincial Hospital, Hefei, China.

${ }^{9}$ Rheumatology Unit, University of Pisa, Pisa, Italy.

${ }^{10}$ Department of Rheumatology, Strasbourg University Hospital, Université de Strasbourg, CNRS, Strasbourg, France.

${ }^{11}$ Department of Clinical Immunology \& Rheumatology, Christian Medical College \& Hospital, Vellore, India.

${ }^{12}$ Clinic of Rheumatology, Department of Medical and Biological Sciences, University Hospital "Santa Maria della Misericordia", Udine, Italy.

${ }^{13}$ Department of Internal Medicine and Medical Specialties, Rheumatology Clinic, Sapienza University of Rome, Rome, Italy.

${ }^{14}$ Immunology and Rheumatology Department, Instituto Nacional de Ciencias Médicas y Nutrición Salvador Zubirán. México City, Mexico.

${ }^{15}$ Department of Rheumatology and Clinical Immunology, University Medical Center Utrecht, Utrecht, The Netherlands.

${ }^{16}$ Department of Medicine, Federal University of Espírito Santo, Vitória, Brazil. 
17 Department of Medicine, Solna, Unit of Experimental Rheumatology, Karolinska Institutet, and Karolinska University Hospital, Stockholm, Sweden.

${ }^{18}$ Service de Médecine Interne 2, Hôpital Lariboisière, Université Paris VII, Assistance Publique-Hôpitaux de Paris, 2, Paris, France.

${ }^{19}$ Rheumatology Unit, Department of Medicine, University of Perugia, Perugia, Italy.

${ }^{20}$ Department of Rheumatology, University Medical Centre, Ljubljana, Slovenia.

${ }^{21}$ Centre for Rheumatology, Division of Medicine, University College London, London, UK.

22 Department of Internal Medicine, Hospital Vall d'Hebron, Barcelona, Spain.

${ }^{23}$ Department of Rheumatology, School of Medicine, The University of Western Australia, Crawley, Australia.

${ }^{24}$ Seoul St. Mary's Hospital, The Catholic University of Korea, Seoul, South Korea.

${ }^{25}$ Rheumatology, Department of Medical Sciences, Uppsala University, Uppsala, Sweden.

${ }^{26}$ Division of Rheumatology, Kanazawa University Hospital, Kanazawa, Ishikawa, Japan.

${ }^{27}$ Clinical Unit of Rheumatology, University of I'Aquila, School of Medicine, L'Aquila, Italy.

${ }^{28}$ Rheumatology Department, Brest University Hospital, Brest, France.

${ }^{29}$ Centre for Experimental Medicine and Rheumatology, Queen Mary University of London, London, UK.

${ }^{30}$ Hals-Nasen-Ohrenklinik und Poliklinik, Technische Universität München, München, Germany.

${ }^{31}$ Department of Rheumatology \& Clinical Immunology, University of Groningen, University Medical Center Groningen, Groningen, The Netherlands.

${ }^{32}$ Department of Clinical Science, University of Bergen; and Department of Rheumatology, Haukeland University Hospital, Bergen, Norway.

${ }^{33}$ Department of Internal Medicine, Hospital Ramón y Cajal, Madrid, Spain.

${ }^{34}$ Division of Rheumatology, Allergy and Immunology Winthrop-University Hospital, Stony Brook University School of Medicine, Mineola, NY, USA.

${ }^{35}$ Rheumatology Department, Kasr Al Ainy School of Medicine, Cairo University, Cairo, Egypt.

${ }^{36}$ Department of Rheumatology, Teaching hospital and University of Montpellier, Montpellier, France.

${ }^{37}$ German Hospital, Buenos Aires, Argentina.

${ }^{38}$ IRCCS Galeazzi Orthopedic Institute, Milan, Italy.

${ }^{39}$ Hospital Privado Universitario de Córdoba, Institute University of Biomedical Sciences University of Córdoba (IUCBC), Cordoba, Argentina.

${ }^{40}$ Federal University of São Paulo, Sao Paulo, Brazil.

${ }^{41}$ Department of Medicine, University of Barcelona, Barcelona, Spain.

*The members of the EULAR-SS Task Force Big Data Consortium are listed in Appendix 1

Supported by Grants Fondo de Investigaciones Sanitarias (MRC, INT15/00085) and "Ajut per a la Recerca Josep Font" (PBZ, Hospital Clinic-Barcelona 2012)

Address reprint requests to: Dr. Manuel Ramos-Casals, Servei de Malalties Autoimmunes Sistèmiques, Hospital Clínic, C/Villarroel, 170, 08036-Barcelona, Spain. Phone: 34-93-2275774.

FAX: 34-93-2271707.e-mail: mramos@clinic.ub.es

\section{WORD COUNT: 3300}




\section{ABSTRACT}

OBJECTIVES. To analyse the influence of geolocation and ethnicity on the clinical presentation of primary Sjögren syndrome (SjS) at diagnosis.

METHODS. The Big Data Sjögren Project Consortium is an international, multicentre registry designed in 2014. By January 2016, 20 centres from five continents were participating. Multivariable logistic regression analyses were performed.

RESULTS. We included 7748 (93\%) women and 562 (7\%) men, with a mean age at diagnosis of primary SjS of 53 years. Ethnicity data was available for 7884 (95\%) patients: 6174 (78\%) patients were White, 1066 (14\%) Asian, 393 (5\%) Hispanic, 104 (1\%) Black/African American and 147 (2\%) had other ethnicities. SjS was diagnosed a mean of 7 years earlier in Black/African American compared with White patients, the female:male ratio was highest in Asian patients (27:1) and lowest in Black/African American patients (7:1); the prevalence of sicca symptoms was lowest in Asian patients; a higher frequency of positive salivary biopsy was found in Hispanic and White patients. A north-south gradient was found with respect to a lower frequency of ocular involvement in northern countries for dry eyes and abnormal ocular tests in Europe (OR 0.46 and 0.44 , respectively) and Asia (OR 0.18 and 0.49 ,respectively) compared with southern countries. Higher frequencies of ANA were reported in northern countries in America $(\mathrm{OR}=1.48)$ and Asia $(\mathrm{OR}=3.80)$ while, in Europe, northern countries had lowest frequencies of ANA (OR=0.67) and Ro/La (OR=0.69). CONCLUSIONS. This study provides the first evidence of a strong influence of geolocation and ethnicity on the phenotype of primary SjS at diagnosis.

KEY WORDS: primary Sjögren syndrome, ethnicity, geoepidemiology, salivary gland biopsy, Ro/La autoantibodies 


\section{INTRODUCTION}

Primary Sjögren syndrome (SjS) is a systemic autoimmune disease that mainly targets the exocrine glands, leading to dryness of the main mucosal surfaces.[1] The histological hallmark is focal lymphocytic infiltration of the targeted organs, and the key immunological markers include ANA (the most frequently detected), anti-Ro/SjS-A (the most specific) and cryoglobulins and hypocomplementaemia (the main prognostic markers).[1] SjS overwhelmingly affects middle-aged women, and its frequency varies widely according to study designs and the classification criteria used. More recent studies using the 2002 American-European classification criteria[2] have reported an incidence of 3-11 cases per 100,000 persons and a prevalence of between 0.01 and $0.72 \% .[3,4]$

The influence of ethnicity on the phenotypic expression of systemic autoimmune diseases has been suggested by various studies, especially in systemic lupus erythematosus (SLE), which has been reported as being more frequent and having less favourable outcomes in non-White populations. $[5,6]$ With respect to the influence of geographical factors, a potential north-south gradient in the frequency of autoimmune diseases has been suggested. $[7,8]$ In primary SjS, there is no information on the influence of ethnicity or geolocation on the phenotypic expression of the disease. Only one recent study, in the general population of Greater Paris,[4] has evaluated the influence of ethnicity on the frequency of primary SjS, and this found a two-fold higher prevalence in patients with non-European backgrounds compared with those with a European background.

The objective of this study was to determine the influence of geolocation and ethnicity on the clinical presentation of primary SjS at diagnosis in a large international cohort of patients. 


\section{METHODS}

\section{Patients}

The Big Data Sjögren Project Consortium is an international, multicentre registry designed in 2014 to take a "high-definition" picture of the main features of primary SjS at diagnosis by merging international SjS databases. International experts from the EULAR-SjS Task Force were invited to participate. Inclusion criteria were the fulfilment of the 2002 classification criteria.[2]; in addition, a letter was sent to the corresponding authors of manuscripts published in the last two years in Pubmed that included clinical data on at least 50 patients with primary SjS, inviting them to join the study. Exclusion criteria for considering SjS as a primary disease were chronic HCV/HIV infections, previous lymphoproliferative processes, and associated systemic autoimmune diseases. Diagnostic tests for SjS (ocular tests, oral tests and salivary gland biopsy) were carried out according to the recommendations of the European Community Study Group.[9] The study was approved by the Ethics Committee of the Coordinating Centre (Hospital Clinic, Barcelona, Spain, registry HCB/2015/0869).

Disease diagnosis was defined as the time when the attending physician confirmed fulfilment of the 2002 criteria. At this time, the main features of the disease were retrospectively collected and analysed (age, gender, ethnicity, country of residence, fulfilment of the 2002 criteria items, antinuclear antibodies, rheumatoid factor, C3 and C4 levels, and cryoglobulins). By January 2016, the participant centres had included 8417 patients from 20 countries in five continents. Further confirmation was made by excluding cases in which fulfilment of the 2002 criteria could not be directly ensured according to the data provided (lack of information about items IV and VI-salivary biopsy and Ro/La autoantibodies).

Patients were classified according to the geolocation of the country of the diagnosing hospital. Patients were first classified by continent, with an additional north-south subclassification according to latitude in continents including patients from > 1 country; the subclassification of the latitudes is not standard and was adapted to the geolocation of the countries included in the registry: latitude $>$ or $<50^{\prime} \mathrm{N}$ in Europe, equator $>$ or $<$ in America and latitude $>$ or $<30^{\prime} \mathrm{N}$ in Asia). Ethnicity was classified retrospectively (asking the patient or relatives if necessary) according to the OMB 
Standards for Maintaining, Collecting, and Presenting Federal Data on Race and Ethnicity of the FDA,[10] using the following categories and definitions:

- Asian. A person having origins in any of the original peoples of the Far East, Southeast Asia, or the Indian subcontinent, including, for example, Cambodia, China, India, Japan, Korea, Malaysia, Pakistan, the Philippine Islands, Thailand, and Vietnam.

- Black or African American. A person having origins in any of the black racial groups of Africa.

- Hispanic or Latino. A person of Cuban, Mexican, Puerto Rican, South or Central American, or other Spanish culture or origin, regardless of race. However, Spanish patients from Spain were included in the White definition.

- White. A person having origins in any of the original peoples of Europe, the Middle East, or North Africa.

- Others. Native Hawaiian or Other Pacific Islander; defined as a person having origins in any of the original peoples of Hawaii, Guam, Samoa, or other Pacific Islands; American Indian or Alaska Native, defined as a person having origins in any of the original peoples of North and South America (including Central America), and who maintains tribal affiliation or community attachment; and patients with one or more racial designations.

\section{Statistical analysis}

Descriptive data are presented as mean and standard deviation (SD) for continuous variables and numbers and percentages (\%) for categorical variables. The prevalence of a specific feature is stated as the number of cases with that feature/number of cases in which the feature was detailed. The Chi-square test was used to study categorical features at diagnosis according to geolocation (continent) and ethnic groups. One-way ANOVA tests were used to compare the mean age at diagnosis. The following subanalyses were made: i) geolocation of countries by latitude (north vs. south) in Europe (latitude $>$ or $<50^{\prime} \mathrm{N}$ ), America (equator $><$ ) and Asia (latitude $>$ or $<30^{\prime} \mathrm{N}$ ); ii) Asian patients and country of residence (Asian vs. non-Asian countries); iii) Hispanic patients and country of residence (Latin American vs. other countries). Clustered bar charts 
were constructed to compare ethnic clusters according to fulfilment of the 2002 criteria items and the baseline immunological profile.[11] P-values were adjusted for multiple comparisons using the false discovery rate (FDR) correction. [12] Multivariable logistic regression analyses adjusted for ethnicity, age at diagnosis and gender were performed to study the association between geolocation with diagnostic tests for SjS and immunological markers at diagnosis. To handle missing data due to non-evaluated diagnostic tests for SjS or non-performed immunological markers, "available case analysis" was assumed for the comparisons according to geolocation and ethnic groups. The missing data pattern shows that most variables had low percentages of missing data (see online supplementary Figure S1). All significance tests were twotailed and values of $p<0.05$ were considered significant. P-values were adjusted for multiple comparisons using the false discovery rate (FDR) correction. All analyses were conducted using the R V.3.2.3 for Windows statistical software package (https://www.R-project.org/.). 


\section{RESULTS}

\section{a) Baseline characterization}

Of the 8417 patients originally included in the database, 107 were excluded in the refinement process (lack of information about items IV and/or VI). The baseline characteristics of the final cohort (8310 patients) are summarized in Table 1 . The cohort included 7748 (93\%) women and 562 (7\%) men (female: male ratio, 14:1), with a mean age at diagnosis of primary SjS of 53.2 (SD 14.2) years. The frequencies of fulfilment of the 2002 classification criteria items were $92 \%$ for dry eye (item I), 93\% for dry mouth (item II), 86\% for abnormal ocular tests (item III), 89\% for positive minor salivary gland biopsy (item IV), $80 \%$ for abnormal oral diagnostic tests (item V) and $75 \%$ for positive anti-Ro/La antibodies (item VI). The frequency of immunological markers at diagnosis was: positive ANA in $81 \%$ of patients, positive RF in $49 \%$, low C3 levels in $14 \%$, low C4 levels in $13 \%$ and positive serum cryoglobulins in $7.5 \%$ of patients.

\section{b) Geolocation}

The main results on geolocation (continent and subareas classified by latitude) are summarized in Table 1. Patients came mainly from Europe $(n=6045)$, America $(n=1134)$ and Asia ( $n=940$ ) (see online supplementary Table S1). Table 2 compares the main geolocational features classified according to latitude in these three continents. Logistic regression analysis adjusted by ethnicity, age at diagnosis and gender showed that northern European patients (latitude $>50^{\prime} \mathrm{N}$ ) had a lower frequency of ocular dryness (OR $0.46,95 \% \mathrm{Cl} 0.37$ to 0.57 ), abnormal ocular tests (OR $0.44,95 \% \mathrm{Cl} 0.37$ to 0.53), ANA (OR $0.67,95 \% \mathrm{Cl} 0.58$ to 0.78 ), low C3 levels (OR $0.76,95 \% \mathrm{Cl} 0.62$ to 0.93 ) and Ro/La autoantibodies (OR $0.69,95 \% \mathrm{Cl} 0.60$ to 0.79 ) and a higher frequency of abnormal oral tests (OR 2.12, 95\% Cl 1.71 to 2.64 ) and RF (OR $1.62,95 \% \mathrm{Cl} 1.41$ to 1.86 ) compared with southern European patients. North American patients had a lower frequency of positive salivary biopsy (OR $0.44,95 \% \mathrm{Cl} 0.20$ to 0.88 ), and a higher frequency of ANA (OR 1.48, 95\% Cl 1.05 to 2.09), RF (OR 2.04, 95\% Cl 1.49 to 2.82), Ro/La autoantibodies (OR 4.10,95\%Cl 2.93 to 5.78) and low C4 levels (OR 6.94, 95\%Cl 3.17 to 18.32 ) compared with South American patients. Northern Asian patients had a lower frequency of dry mouth (OR $0.30,95 \% \mathrm{Cl} 0.19$ to 0.46 ), dry eyes (OR $0.18,95 \% \mathrm{Cl}$ 0.12 to 0.28 ), abnormal ocular tests (OR $0.49,95 \% \mathrm{Cl} 0.30$ to 0.79 ) and positive salivary 
biopsy (OR $0.42,95 \% \mathrm{Cl} 0.26$ to 0.68 ), and a higher frequency of ANA (OR $3.80,95 \% \mathrm{Cl}$ 2.54 to 5.73 ) and Ro/La autoantibodies (OR $2.78,95 \% \mathrm{Cl} 1.89$ to 4.09 ), compared with southern Asian patients.

\section{c) Ethnicity}

Ethnicity data was available for 7884 (95\%) patients of the total cohort: 6174 (78.3\%) patients were classified as White, 1066 (13.5 \%) as Asian, 393 (5\%) as Hispanic, 104 (1.3\%) as Black/African American patients and 147 (1.9\%) as other ethnicities (Table 1). Patients from European centres were overwhelmingly White compared with those from American centres ( $96 \%$ vs. 52\%, p<0.001); Supplementary Table $\$ 2$ summarizes the main features of White patients compared with patients of other ethnicities. Table 3 shows the main features at presentation according to ethnicity: the highest percentage of males was in Black/African American patients and the lowest in Asian patients; the youngest age at diagnosis was in Black/African American patients and the oldest in White patients; the lowest frequency of sicca symptoms was in Asian patients and the highest in other ethnicities; the lowest frequency of abnormal diagnostic tests was in patients of other ethnicities and the highest in Hispanic patients; and the highest frequency of Ro/La autoantibodies was in Asian patients and the lowest in patients of other ethnicities. Figure 1 includes clustered bar charts for the percentage of fulfilment of the six items of the 2002 classification criteria, and Figure 2 the percentage of abnormal results in the immunological profile according to ethnicity. The potential effect of geolocation in patients classified in the same ethnic group was analysed in two sub-studies. Asian patients diagnosed in non-Asian countries had a higher frequency of dry mouth $(p=0.007)$ and dry eye $(p=0.003)$ compared with native patients (see online supplementary Figure S2). Hispanic patients living outside Latin American countries had a lower frequency of abnormal salivary biopsy $(p=0.001)$ and positive RF $(p=0.008)$, and a higher frequency of low C3 levels $(p<0.001)$ compared with native patients (see online supplementary Figure S3). 


\section{DISCUSSION}

The etiopathogenesis of primary SjS is unknown. The most frequently proposed hypothesis is based on the effect of multiple, mainly unknown, environmental factors affecting an individual with a specific genetic susceptibility. Geoepidemiological and ethnic studies may help elucidate the complex combination of genes and environment in systemic autoimmune diseases.[7] The most relevant studies have been carried out in SLE: US studies have reported a 2-3-fold higher incidence and prevalence of disease rates in African American patients, UK studies up to 8-fold higher rates in AfroCaribbean and Asian patients, and other studies have found higher prevalences in Native American Indians, Pacific People and Aborigines compared with European populations.[13] In systemic sclerosis, geoepidemiological studies have revealed a higher frequency in the US and Australia than in Europe and Asia,[7] while in primary SjS, a two-fold higher prevalence in patients with non-European backgrounds has recently been reported.[4]

Ethnicity also influences the phenotypic expression of autoimmune diseases, including the clinical course and outcomes. In SLE, the LUMINA project (Lupus in Minorities: Nature versus Nurture) found that African American and Hispanic American SLE patients tend to develop the disease earlier and present with more-severe disease.[14] In systemic sclerosis, a higher mortality rate has been reported in African American populations compared with White populations, [7] while in systemic vasculitis, nonEuropean patients with ANCA-vasculitis also showed more severe disease and higher damage scores.[15]

Until now, no studies have focused on the influence of geoepidemiology and ethnicity on the phenotypic expression of primary SjS. We evaluated these factors in the largest reported series of patients with primary SjS fulfilling the 2002 criteria from 20 countries across five continents. We found significant variations between ethnic groups. The disease was diagnosed a mean of seven years earlier in Black/African American patients compared with White patients, a trend also reported by Maldini et al. in the Parisian multi-ethnic cohort.[4] The female: male ratio also varied significantly, with the highest ratio (27:1) in Asian patients and the lowest (7:1) in Black/African American patients. The prevalence of sicca symptoms at diagnosis also varied significantly: the lowest frequencies were in Asian patients, a finding that has 
been related to cultural differences in previous studies.[16]

This is the first study to analyse the influence of ethnicity on the results of SjS diagnostic tests included in the current classification criteria. Hispanic patients had the highest rates of abnormal results and higher frequencies of subjective dryness symptoms. In contrast, patients of other ethnicities had the lowest rates of abnormal results but higher frequencies of sicca symptoms. This might suggest that ethnicity may influence the results of the objective diagnostic tests for dry eyes and mouth in primary SjS patients, with Hispanic and White patients being more likely to have abnormal results compared with other ethnicities. With respect to the frequency of fulfilment of the histopathological criteria (salivary biopsy showing Chisholm-Mason grade 3 or 4), we found a pattern of ethnic association similar to that observed for objective tests for dryness, with a higher frequency of positive salivary biopsy in Hispanic and White patients compared with the other ethnicities; Maldini et al.[4] reported a similar, although not significant, trend in the multi-ethnic Paris cohort. The influence of ethnicity on the phenotypic expression of primary SjS at diagnosis could be driven by immunogenetic differences. Maldini et al.[4] found a younger age at diagnosis and an increased frequency of polyclonal hypergammaglobulinaemia and positive Ro/La antibodies in non-European patients, a reasonable association since immunopositive SjS patients are often diagnosed earlier.[17] We have confirmed this association: patients from ethnic groups with the highest frequencies of positive antiRo antibodies (Asian, Hispanic and Black/African American ) also had the youngest ages at diagnosis; a similar trend was observed for anti-La antibodies, except for Black/African American patients, who had the second-lowest frequency of all ethnic groups. Ro/La immunogenicity has traditionally been linked with genetic factors, mainly with specific HLA class II alleles,[18] and a joint contribution of HLA-DR and DQ alleles has been suggested as relevant for the development of antibodies against Ro/La autoantigens.[19] Most SjS patients share a common allele (DQA1*0501) across racial and ethnic boundaries.[20] However, Kang et al.[21] found significant differences in the frequency of some HLA-DR haplotypes (a higher frequency of DRB3 in Caucasians and DRB4 in Japanese, and a lower frequency of DPB1 in Chinese patients). Future immunogenetic studies in primary SjS should evaluate the influence of ethnicity on the results, searching for possible immunogenetic differences. 
A potential north-south autoimmune gradient, with rates seeming to increase according to distance from the Equator, has been suggested in the prevalence and incidence of several autoimmune diseases including type 1 diabetes mellitus, multiple sclerosis, and inflammatory bowel disease.[7,22-25] Little geoepidemiological data is available for systemic autoimmune diseases, and there are no data on primary SjS. The present study found some interesting results after comparing northern vs. southern countries in the three continents for which data from more than one country are available (Europe, America and Asia). A north-south gradient was confirmed with respect to a lower frequency of ocular involvement and a higher frequency of cryoglobulinemic-related tests (cryoglobulins and hypocomplementaemia) in northern compared with southern countries. The gradient was different in Europe with respect to the other components of primary SjS. For salivary gland involvement, the highest rates of abnormal results (including biopsy) in Europe were found in patients from northern countries, while in America and Asia the highest rates were reported in patients from southern countries. A similar gradient was observed with respect to autoantibodies (ANA, Ro, La): the highest frequencies in America and Asia were reported in northern countries while in Europe, the highest frequencies were reported in southern countries. These results suggest, for the first time, that geolocation may influence the phenotypic expression of primary SjS at diagnosis, including significant geoepidemiological variations in the prevalence of dryness, the frequency of abnormal diagnostic tests and the positivity of the main immunological markers.

We also analysed the influence of geoepidemiological migration on the phenotypic expression of primary SjS at diagnosis by comparing ethnic migrant and native populations. Interestingly, Asian patients diagnosed with primary SjS in non-Asian countries (overwhelmingly in Europe and the US) had a higher frequency of sicca symptoms than Asian patients diagnosed in Asian countries. With respect to Hispanic patients diagnosed outside Latin America, differences were found in diagnostic tests, with a lower frequency of positive salivary biopsy, a lower frequency of positive RF and a higher frequency of low C3 values. No other studies have analysed this, although the study by Maldini et al.[4] reported a differing clinical and immunological pattern of SjS expression in French patients with a non-European background. 
The results, however, should be interpreted with caution, and some limitations should be pointed out. Large studies may detect some differences which, although statistically significant, may not be relevant clinically, with further studies being necessary to confirm their clinical relevance. In addition, the predominant presence of European patients (due to the origin of the project in the EULAR SS Group) could limit the generalization of the results, due to the small size of some ethnic subpopulations, such as Black African American patients. With respect to the study design, although studies comparing relative frequencies of clinical features should, ideally, be population based, our study was designed according to a "Data Sharing" approach, which is currently considered an alternative way of international scientific collaboration, especially in diseases with a low prevalence [27]. Since the participant centers are mainly tertiary university centers that are considered the referral center in their corresponding cities (and in most cases, in their countries), the magnitude of the selection bias may vary between the 20 countries involved in the study and this could have an impact on the results (Supplementary Figure S4 summarizes the size of each cohort classified per city), as may differing medical practices across regions (availability of diagnostic tests included in the 2002 criteria); in fact, we found a negative correlation between the percentage of biopsied patients and the percentage of Ro/La positive patients in each center $(R=-0.55)$ (Supplementary Figure S5). Other sources of heterogeneity may include the assays used by the different centers, although all are commercial tests and more than $80 \%$ used the same technique (ELISA) to test for Ro/La autoantibodies and ANA were overwhelmingly ( $>95 \%$ ) tested for by indirect immunofluorescence), and the missing data for some variables (Supplementary Figure S1).

In summary, this study provides the first evidence for a strong influence of geolocation and ethnicity on the phenotype of primary SjS at diagnosis. Genetic and environmental factors probably contribute to phenotypic variance in SjS, and a recent study has attributed $54 \%$ of the predisposition to developing the disease to familial transmission (heritability plus shared environmental factors) and $46 \%$ to non-shared environmental factors.[26] Geoepidemiology and ethnicity should be considered as key variables that should be analysed in multi-ethnic studies of patients with primary SjS. 


\section{ACKNOWLEDGMENTS}

The authors wish to thank David Buss for his editorial assistance.

\section{COMPETING INTEREST}

None declared.

\section{APPENDIX 1}

Members of the EULAR-SS Task Force Big Data Consortium:

\section{a) Members of the EULAR-SS Task Force}

P. Brito-Zerón, C. Morcillo (Autoimmune Diseases Unit, Department of Medicine, Hospital CIMA- Sanitas, Barcelona, Spain); P. Brito-Zerón, I. García-Sánchez, H. Gueitasi, A. Bové, M. Ramos-Casals (Sjögren Syndrome Research Group (AGAUR), Laboratory of Autoimmune Diseases Josep Font, IDIBAPS-CELLEX, Department of Autoimmune Diseases, ICMiD, University of Barcelona, Hospital Clínic, Barcelona, Spain); N. Acar-Denizli (Department of Statistics, Faculty of Science and Letters, Mimar Sinan Fine Arts University, Istanbul, Turkey); M. Zeher, Ildike-Fanny Horvath (Division of Clinical Immunology, Faculty of Medicine, University of Debrecen, Debrecen, Hungary); A. Rasmussen, K. Sivils, H. Scofield (Arthritis and Clinical Immunology Research Program, Oklahoma Medical Research Foundation, Oklahoma City, OK, USA); R. Seror, X. Mariette (Center for Immunology of Viral Infections and Autoimmune Diseases, Assistance Publique - Hôpitaux de Paris, Hôpitaux Universitaires Paris-Sud, Le Kremlin-Bicêtre, Université Paris Sud, INSERM, Paris, France Paris, France); E. Theander, T. Mandl (Department of Rheumatology, Malmö University Hospital, Lund University, Lund, Sweden); X. Li (Department of Rheumatology and Immunology, Anhui Provincial Hospital, China); C. Baldini (Rheumatology Unit, University of Pisa, Pisa, Italy); J.E. Gottenberg (Department of Rheumatology, Strasbourg University Hospital, Université de Strasbourg, CNRS, Strasbourg, France); D. Danda, P. Sandhya (Department of Clinical Immunology \& Rheumatology, Christian Medical College \& Hospital, Vellore, India); L. Quartuccio, L. Corazza, S De Vita (Clinic of Rheumatology, Department of Medical and Biological Sciences, University Hospital "Santa Maria della Misericordia", Udine, Italy); R. Priori, E. Bartoloni (Department of Internal Medicine and Medical Specialties, Rheumatology Clinic, Sapienza University of Rome, Italy); G. Hernandez-Molina, J. Sánchez-Guerrero (Immunology and Rheumatology Department, Instituto Nacional de Ciencias Médicas y Nutrición Salvador Zubirán. México City, Mexico); A.A. Kruize, E. van der Heijden (Department of Rheumatology and Clinical Immunology, University Medical Center Utrecht, Utrecht, The Netherlands); V. Valim (Department of Medicine, Federal University of Espírito Santo, Vitória, Brazil); M. Kvarnstrom, M. Wahren-Herlenius (Department of Medicine, Solna, Unit of Experimental Rheumatology, Karolinska Institutet, and Karolinska University Hospital, Stockholm); D. Sene (Service de Médecine Interne 2, Hôpital Lariboisière, Université Paris VII, Assistance Publique-Hôpitaux de Paris, 2, Paris, France); R. Gerli (Rheumatology Unit, Department of Medicine, University of Perugia, Italy); S. Praprotnik (Department of Rheumatology, University Medical Centre, Ljubljana, Slovenia); D. Isenberg (Centre for Rheumatology, Division of Medicine, University College London, UK); R. Solans (Department of Internal Medicine, Hospital Vall d'Hebron, Barcelona, Spain); M. Rischmueller, S. Downie-Doyle (Department of Rheumatology, School of Medicine, The University of Western Australia, Crawley, Australia); S-K. Kwok, S-H. Park (Seoul St. Mary's Hospital, The Catholic University of Korea, Seoul); G. Nordmark (Rheumatology, Department of Medical Sciences, Uppsala University, Uppsala, Sweden); Y. Suzuki, M. Kawano (Division of Rheumatology, Kanazawa University Hospital , Kanazawa , Ishikawa , Japan); R. Giacomelli, F. Carubbi (Clinical Unit of Rheumatology, University of I'Aquila, School 
of Medicine, L'Aquila, Italy); V. Devauchelle-Pensec, A. Saraux (Rheumatology Department, Brest University Hospital, Brest, France); M. Bombardieri, E. Astorri (Centre for Experimental Medicine and Rheumatology, Queen Mary University of London, UK); B. Hofauer (Hals-Nasen-Ohrenklinik und Poliklinik, Technische Universität München, München, Germany); H. Bootsma, A. Vissink (Department of Rheumatology \& Clinical Immunology, University of Groningen, University Medical Center Groningen, the Netherlands); J.G. Brun, D. Hammenfors (Department of Rheumatology, Haukeland University Hospital, Bergen, Norway); G. Fraile (Department of Internal Medicine, Hospital Ramón y Cajal, Madrid, Spain); S. E. Carsons (Division of Rheumatology, Allergy and Immunology Winthrop-University Hospital, Stony Brook University School of Medicine, Mineola, NY, USA); T. A. Gheita, (Rheumatology Department, Kasr Al Ainy School of Medicine, Cairo University, Egypt); H.M. Khalil (Ophthalmology Department, Faculty of Medicine, Beni Suef University, Egypt); J. Morel (Department of Rheumatology, Teaching hospital and University of Montpellier, Montpellier, France); C. Vollenveider (German Hospital, Buenos Aires, Argentina); F. Atzeni (IRCCS Galeazzi Orthopedic Institute, Milan, Italy); S. Retamozo (Hospital Privado Universitario de Córdoba, Institute University of Biomedical Sciences University of Córdoba, Córdoba, Argentina; V. Moça Trevisano (Federal University of São Paulo, Sao Paulo, Brazil); B. Kostov, A. SisóAlmirall (Primary Care Research Group, IDIBAPS, Centre d'Assistència Primària ABS Les Corts, CAPSE, Barcelona, Spain).

\section{b) Members of the French ASSESS Cohort}

J. Sibilia (Rheumatology Centre National de Référence des Maladies Auto-Immunes Rares, Institut National de la Santé et de la Recherche Médicale UMRS_1109, Fédération de Médecine Translationnelle de Strasbourg, Strasbourg University Hospital, Université de Strasbourg, Strasbourg, France); C. Miceli-Richard, G. Nocturne (Rheumatology, Bicetre Hospital, Institut National de la Santé et de la Recherche Médicale U-1012, Université Paris Sud, Assistance Publique des Hôpitaux de Paris, Paris, France); J. Benessiano (Centre de Ressources Biologiques, Bichat Hospital, Assistance Publique des Hôpitaux de Paris, Paris, France); P. Dieude (Rheumatology, Bichat Hospital, Assistance Publique des Hôpitaux de Paris, Paris, France); J-J. Dubost (Rheumatology, Clermont-Ferrand Hospital, ClermontFerrand, France); A-L. Fauchais (Internal Medicine, Limoges Hospital, Limoges, France); V. Goeb (Rheumatology, Amiens University Hospital, Amiens, France); E. Hachulla (Pierre Yves Hatron, Internal Medicine, Lille University Hospital, Lille, France); C. Larroche (Internal Medicine, Avicenne Hospital, Assistance Publique des Hôpitaux de Paris, Bobigny, France); V. Le Guern, X. Puéchal (Internal Medicine, Cochin Hospital, Assistance Publique des Hôpitaux de Paris, Paris, France); J. Morel (Rheumatology, Montpellier University Hospital, Montpellier, France); A. Perdriger (Rheumatology, Rennes University Hospital, Rennes, France); S. Rist, Rheumatology, Orléans Hospital, Orléans, France; O. Vittecoq (Rheumatology, Rouen University Hospital, Rouen, France); P. Ravaud (Centre of Clinical Epidemiology, Hotel Dieu Hospital, Assistance Publique des Hôpitaux de Paris, Institut National de la Santé et de la Recherche Médicale U378, University of Paris Descartes, Faculty of Medicine, Paris, France).

\footnotetext{
c) Members of the Spanish GEAS Cohort (SS Study Group, Autoimmune Diseases Study Group GEAS, Spanish Society of Internal Medicine SEMI):

B. Díaz-López (Department of Internal Medicine, Hospital Universitario Central de Asturias, Oviedo), A. Casanovas, (Department of Internal Medicine, Hospital Parc Taulí, Sabadell), L. Pallarés (Department of Internal Medicine, Hospital Son Espases, Palma de Mallorca), M. López-Dupla (Department of Internal Medicine, Hospital Joan XXIII, Tarragona), R. Pérez-Alvarez (Department of Internal Medicine, Hospital do Meixoeiro, Vigo), M. Ripoll (Department of Internal Medicine, Hospital Infanta Sofía, Madrid), B. Pinilla (Department of Internal Medicine, Hospital Gregorio
} 
Marañón, Madrid), M. Akasbi (Department of Internal Medicine, Hospital Infanta Leonor, Madrid), B. Maure (Department of Internal Medicine, Complejo Hospitalario Universitario, Vigo), E. Fonseca (Department of Internal Medicine, Hospital de Cabueñes, Gijón), J. Canora (Department of Internal Medicine, Hospital Universitario de Fuenlabrada, Madrid), G de la Red (Department of Internal Medicine, Hospital Espíritu Santo, Barcelona), A.J. Chamorro (Department of Internal Medicine, Complejo Hospitalario de Ourense, Ourense), I. Jiménez-Heredia (Department of Internal Medicine, Hospital de Manises, Valencia, Spain), P. Fanlo (Complejo Universitario de Navarra), P. Guisado-Vasco (Hospital Quirón, Madrid), M. Zamora (Hospital Virgen de las Nieves, Granada). 


\section{REFERENCES}

1. Ramos-Casals M, Brito-Zerón P, Sisó-Almirall A, et al. Primary Sjogren syndrome. BMJ. 2012;344:e3821.

2. Vitali C, Bombardieri S, Jonsson R, et al. Classification criteria for Sjögren's syndrome: a revised version of the European criteria proposed by the AmericanEuropean Consensus Group. Ann Rheum Dis. 2002;61(6):554-58.

3. Qin B, Wang J, Yang Z, et al. Epidemiology of primary Sjogren's syndrome: a systematic review and meta-analysis. Ann Rheum Dis. 2015;74(11):1983-89.

4. Maldini C, Seror R, Fain O, et al. Epidemiology of primary Sjögren's syndrome in a French multiracial/multiethnic area. Arthritis Care Res (Hoboken). 2014;66(3):454-63.

5. Gonzalez LA, Toloza SMA, McGwin GJ, et al. Ethnicity in systemic lupus erythematosus (SLE): its influence on susceptibility and outcomes. Lupus. 2013;22(12):1214-24.

6. Gonzalez LA, Toloza SMA, Alarcon GS. Impact of race and ethnicity in the course and outcome of systemic lupus erythematosus. Rheum Dis Clin North Am. 2014;40(3):433-54, vii - viii.

7. Shapira Y, Agmon-Levin N, Shoenfeld Y. Geoepidemiology of autoimmune rheumatic diseases. Nat Rev Rheumatol. 2010;6(8):468-476.

8. Selmi C. The worldwide gradient of autoimmune conditions. Autoimmun Rev. 2010;9(5):A247-A250.

9. Vitali C, Bombardieri S, Moutsopoulos HM, et al. Preliminary criteria for the classification of Sjögren's syndrome. Results of a prospective concerted action supported by the European Community. Arthritis Rheum. 1993;36(3):340-47.

10. Office of Management and Budget. Revisions to the Standards for the Classification of Federal Data on Race and Ethnicity. Federal Register. 1997;62:58781-90.

11. Wickham H. ggplot2: elegant graphics for data analysis. New York: Springer, 2009.

12. Benjamini Y, Hochberg Y. Controlling the False Discovery Rate: A Practical and Powerful Approach to Multiple Testing. J R Stat Soc Ser B. 1995;57(1):289-300.

13. Borchers AT, Naguwa SM, Shoenfeld $\mathrm{Y}$, et al. The geoepidemiology of systemic 
lupus erythematosus. Autoimmun Rev. 2010;9(5):A277-A287.

14. Uribe AG, McGwin GJ, Reveille JD, et al. What have we learned from a 10-year experience with the LUMINA (Lupus in Minorities; Nature vs. nurture) cohort? Where are we heading? Autoimmun Rev. 2004;3(4):321-29.

15. Sreih AG, Mandhadi R, Aldaghlawi F, et al. ANCA-associated vasculitis in Hispanic Americans: an unrecognized severity. Clin Rheumatol. 2015;34(5):943-48.

16. Zhao Y, Li Y, Wang L, et al. Primary Sjogren syndrome in Han Chinese: clinical and immunological characteristics of 483 patients. Medicine (Baltimore). 2015;94(16):e667.

17. Brito-Zerón $P$, Theander E, Baldini C, et al. Early Diagnosis of primary Sjögren's Syndrome: EULAR-SS Task Force Clinical Recommendations. Expert Rev Clin Immunol. 2016;12(2):137-56.

18. Routsias JG, Tzioufas AG. Autoimmune response and target autoantigens in Sjogren's syndrome. Eur J Clin Invest. 2010;40(11):1026-36.

19. Bolstad Al, Wassmuth R, Haga HJ, et al. HLA markers and clinical characteristics in Caucasians with primary Sjogren's syndrome. J Rheumatol. 2001;28(7):155462.

20. Roitberg-Tambur A, Friedmann A, Safirman C, et al. Molecular analysis of HLA class II genes in primary Sjogren's syndrome. A study of Israeli Jewish and Greek non-Jewish patients. Hum Immunol. 1993;36(4):235-42.

21. Kang HI, Fei HM, Saito I, et al. Comparison of HLA class II genes in Caucasoid, Chinese, and Japanese patients with primary Sjogren's syndrome. J Immunol. 1993;150(8 Pt 1):3615-23.

22. Okada $\mathrm{H}$, Kuhn $\mathrm{C}$, Feillet $\mathrm{H}$, et al. The "hygiene hypothesis" for autoimmune and allergic diseases: an update. Clin Exp Immunol. 2010;160(1):1-9.

23. Bach J-F. The effect of infections on susceptibility to autoimmune and allergic diseases. N Engl J Med. 2002;347(12):911-20.

24. Youinou P, Pers J-O, Gershwin ME, et al. Geo-epidemiology and autoimmunity. J Autoimmun. 2010;34(3):J163-J167.

25. Shoenfeld $\mathrm{Y}$, Selmi C, Zimlichman E, et al. The autoimmunologist: geoepidemiology, a new center of gravity, and prime time for autoimmunity. $J$ Autoimmun. 2008;31(4):325-30. 
26. Kuo C-F, Grainge MJ, Valdes AM, et al. Familial Risk of Sjögren's Syndrome and Co-aggregation of Autoimmune Diseases in Affected Families: A Nationwide Population Study. Arthritis Rheumatol (Hoboken, NJ). 2015;67(7):1904-12.

27. Warren E. Strengthening Research through Data Sharing. N Engl J Med. 2016;375(5):401-3. 
TABLE 1. Baseline characteristics of 8310 patients with primary SjS

\begin{tabular}{|c|c|}
\hline Variable & Patients (\%) \\
\hline Gender (Female) & $7748(93.2)$ \\
\hline Age at diagnosis $(n=8270)$ & $53.2 \pm 14.2$ \\
\hline Dry eye & $7660(92.2)$ \\
\hline Dry mouth & $7700(92.7)$ \\
\hline Abnormal ocular tests & $6228 / 7273(85.6)$ \\
\hline Schirmer's test & $4903 / 6203(79.0)$ \\
\hline Rose bengal score or other ocular dye score & $2460 / 3302(74.5)$ \\
\hline Positive minor salivary gland biopsy & $5305 / 5984(88.7)$ \\
\hline Abnormal oral diagnostic tests & $4843 / 6063(79.9)$ \\
\hline Unstimulated whole salivary flow & $3608 / 4938(73.1)$ \\
\hline Parotid sialography & $702 / 873(80.4)$ \\
\hline Salivary scintigraphy & $2160 / 2578(83.8)$ \\
\hline Positive anti-Ro/La antibodies & $6177 / 8250(74.9)$ \\
\hline Anti-Ro antibodies & $5950 / 8245(72.2)$ \\
\hline Anti-La antibodies & $3599 / 8215(43.8)$ \\
\hline ANA positive & $6292 / 7746(81.2)$ \\
\hline RF positive & $3483 / 7154(48.7)$ \\
\hline C3 low & $912 / 6554(13.9)$ \\
\hline C4 low & $846 / 6540$ (12.9) \\
\hline Positive cryoglobulins & $307 / 4118(7.5)$ \\
\hline \multicolumn{2}{|l|}{ Ethnicity } \\
\hline White & $6174 / 7884(78.3)$ \\
\hline Asian & $1066 / 7884(13.5)$ \\
\hline Hispanic & $393 / 7884(5.0)$ \\
\hline Black/African American & $104 / 7884(1.3)$ \\
\hline Others & $147 / 7884(1.9)$ \\
\hline \multicolumn{2}{|l|}{ Geolocation } \\
\hline Europe & $6045(72.7)$ \\
\hline North $(>50-N)$ & $1393(16.7)$ \\
\hline South $(<509 \mathrm{~N})$ & $4652(56.0)$ \\
\hline America & $1134(13.6)$ \\
\hline North $(>0$ ) & $881(10.6)$ \\
\hline South $(<0$ o $)$ & $253(3.0)$ \\
\hline Asia & $940(11.3)$ \\
\hline North (>30N) & $300(3.6)$ \\
\hline South $(<309 N)$ & $640(7.7)$ \\
\hline Africa & $45(0.6)$ \\
\hline Australia & $146(1.8)$ \\
\hline
\end{tabular}


TABLE 2. Features at diagnosis according to geolocation.

\begin{tabular}{|c|c|c|c|c|c|c|c|c|c|}
\hline \multirow[b]{2}{*}{ Variable } & \multicolumn{3}{|c|}{ EUROPE } & \multicolumn{3}{|c|}{ AMERICA } & \multicolumn{3}{|c|}{ ASIA } \\
\hline & $\begin{array}{c}\text { North }(>509 N) \\
(n=1393)\end{array}$ & $\begin{array}{c}\text { South (<50N) } \\
(n=4652)\end{array}$ & P-value ${ }^{+}$ & $\begin{array}{c}\text { North }(>09) \\
(n=881)\end{array}$ & $\begin{array}{c}\text { South }(<0=) \\
(n=253)\end{array}$ & P-value ${ }^{\ddagger}$ & $\begin{array}{c}\text { North }(>309 N) \\
(n=640)\end{array}$ & $\begin{array}{l}\text { South }(<309 N) \\
(n=300)\end{array}$ & P-value ${ }^{\S}$ \\
\hline Gender (Female) & $1286(92.3)$ & $4311(92.7)$ & 0.741 & $821(93.2)$ & $247(97.6)$ & 0.022 & $623(97.3)$ & $287(95.7)$ & 0.366 \\
\hline Age at diagnosis & $54 \pm 14.7$ & $54 \pm 14$ & 0.997 & $53.9 \pm 14.1$ & $50 \pm 12.6$ & $<0.001$ & $50.2+14.1$ & $43.7 \pm 10.4$ & $<0.001$ \\
\hline Dry eye & $1251(89.8)$ & $4414(94.9)$ & $<0.001$ & $856(97.2)$ & $247(97.6)$ & 0.951 & $428(66.9)$ & $275(91.7)$ & $<0.001$ \\
\hline Dry mouth & $1309(94)$ & $4322(92.9)$ & 0.250 & $867(98.4)$ & $243(96)$ & 0.057 & $496(77.5)$ & $276(92)$ & $<0.001$ \\
\hline Abnormal ocular tests & 933/1175 (79.4) & $3519 / 4024(87.5)$ & $<0.001$ & $664 / 780(85.1)$ & 199/243 (81.9) & 0.333 & $514 / 608(84.5)$ & $244 / 266$ (91.7) & 0.011 \\
\hline Schirmer's test & $884 / 1171(75.5)$ & $2483 / 2972(83.5)$ & $<0.001$ & $499 / 774(64.5)$ & $190 / 243(78.2)$ & $<0.001$ & $452 / 601(75.2)$ & $244 / 266(91.7)$ & $<0.001$ \\
\hline Rose bengal/other ocular dye score & $269 / 479(56.2)$ & $1436 / 1703(84.3)$ & $<0.001$ & $431 / 660(65.3)$ & 77/97 (79.4) & 0.018 & $237 / 350(67.7)$ & NP & NA \\
\hline Positive minor salivary gland biopsy & 1092/1199 (91.1) & $2864 / 3221(88.9)$ & 0.061 & $559 / 655(85.3)$ & $176 / 185(95.1)$ & 0.002 & $282 / 353(79.9)$ & $244 / 270(90.4)$ & 0.001 \\
\hline Abnormal oral diagnostic tests & $893 / 1007(88.7)$ & $2602 / 3365(77.3)$ & $<0.001$ & $566 / 760(74.5)$ & $205 / 243(84.4)$ & 0.005 & $494 / 596(82.9)$ & $3 / 3(100)$ & 1 \\
\hline Unstimulated whole salivary flow & $861 / 995(86.5)$ & $1573 / 2382(66)$ & $<0.001$ & $547 / 748(73.1)$ & $185 / 232(79.7)$ & 0.070 & $375 / 494(75.9)$ & NP & NA \\
\hline Parotid sialography & $41 / 47(87.2)$ & $606 / 738(82.1)$ & 0.572 & 29/33 (87.9) & $1 / 1(100)$ & 1 & 10/17 (58.8) & $1 / 1(100)$ & 1 \\
\hline Salivary scintigraphy & $210 / 224(93.8)$ & $1657 / 2019(82.1)$ & $<0.001$ & $23 / 27(85.2)$ & $70 / 80(87.5)$ & 1 & $169 / 181(93.4)$ & $2 / 2(100)$ & 1 \\
\hline Positive anti-Ro/La antibodies & $955 / 1387(68.9)$ & $3470 / 4640(74.8)$ & $<0.001$ & $679 / 880(77.2)$ & $138 / 246(56.1)$ & $<0.001$ & $572 / 633(90.4)$ & $216 / 280(77.1)$ & $<0.001$ \\
\hline Anti-Ro antibodies & $946 / 1386(68.3)$ & $3328 / 4637(71.8)$ & 0.019 & $631 / 879(71.8)$ & $136 / 246(55.3)$ & $<0.001$ & $550 / 633(86.9)$ & $215 / 280(76.8)$ & 0.001 \\
\hline Anti-La antibodies & $598 / 1379(43.4)$ & $1974 / 4625(42.7)$ & 0.741 & $406 / 880(46.1)$ & $60 / 246(24.4)$ & $<0.001$ & $317 / 628(50.5)$ & $131 / 274(47.8)$ & 0.651 \\
\hline ANA positive & $1020 / 1334(76.5)$ & $3528 / 4259(82.8)$ & $<0.001$ & $672 / 848(79.2)$ & $179 / 248(72.2)$ & 0.039 & $555 / 601(92.3)$ & $216 / 284(76.1)$ & $<0.001$ \\
\hline RF positive & $652 / 1094(59.6)$ & $1931 / 4086(47.3)$ & $<0.001$ & $368 / 834(44.1)$ & $88 / 252(34.9)$ & 0.022 & 295/595 (49.6) & $135 / 248(54.4)$ & 0.366 \\
\hline C3 low & $148 / 806(18.4)$ & $546 / 3798(14.4)$ & 0.008 & $31 / 728(4.3)$ & $8 / 252(3.2)$ & 0.668 & $132 / 601(22)$ & $35 / 212(16.5)$ & 0.201 \\
\hline C4 low & $116 / 741(15.7)$ & $547 / 3850(14.2)$ & 0.416 & 78/726 (10.7) & $6 / 252(2.4)$ & $<0.001$ & $60 / 602(10)$ & $17 / 212(8)$ & 0.651 \\
\hline Positive cryoglobulins & $36 / 237(15.2)$ & $262 / 3449(7.6)$ & $<0.001$ & $3 / 42(7.1)$ & $0 / 108(0)$ & 0.048 & $2 / 124(1.6)$ & $0 / 94(0)$ & 0.724 \\
\hline
\end{tabular}

Adjusted p-values for sixty comparisons with false discovery rate (FDR) correction corresponding to the comparison of European ( $\dagger$ ), American ( $\ddagger)$ and Asian ( $($ ) countries.

NP: Not performed, NA: Not available.

In bold: Statistically significant $(p<0.05)$ variables associated with geolocation in the multivariable logistic regression analysis adjusted for ethnicity, age at diagnosis and gender. 
TABLE 3. Features at diagnosis according to ethnicity groups.

\begin{tabular}{|c|c|c|c|c|c|}
\hline Variable & $\begin{array}{c}\text { White } \\
(n=6174)\end{array}$ & $\begin{array}{c}\text { Asian } \\
(n=1066)\end{array}$ & $\begin{array}{l}\text { Hispanic } \\
(\mathrm{n}=393)\end{array}$ & $\begin{array}{c}\text { Black/African } \\
\text { American }(n=104)\end{array}$ & $\begin{array}{c}\text { Others } \\
(n=147)\end{array}$ \\
\hline Gender (Female) & $5720(92.6)$ & $1028(96.4)$ & $372(94.7)$ & $91(87.5)$ & 141 (95.9) \\
\hline Age at diagnosis & $54.2 \pm 14.2$ & $48.3 \pm 13.3$ & $47.8 \pm 12.9$ & $47.2 \pm 12.4$ & $52.5 \pm 13.7$ \\
\hline Dry eye & $5826(94.4)$ & $817(76.6)$ & $376(95.7)$ & $97(93.3)$ & $144(98)$ \\
\hline Dry mouth & $5775(93.5)$ & $891(83.6)$ & $378(96.2)$ & $99(95.2)$ & $146(99.3)$ \\
\hline Abnormal ocular tests & $4656 / 5354(87)$ & $818 / 953(85.8)$ & $334 / 373(89.5)$ & $65 / 78(83.3)$ & $115 / 146(78.8)$ \\
\hline Schirmer's test & $3525 / 4351(81)$ & $746 / 940(79.4)$ & $301 / 345(87.2)$ & $33 / 52(63.5)$ & $59 / 146(40.4)$ \\
\hline Rose bengal score or other ocular dye score & $1949 / 2564(76)$ & $261 / 387(67.4)$ & $132 / 175(75.4)$ & $20 / 30(66.7)$ & $95 / 140$ (67.9) \\
\hline Positive minor salivary gland biopsy & $3943 / 4406(89.5)$ & $592 / 709(83.5)$ & $258 / 272(94.9)$ & $66 / 81(81.5)$ & $103 / 138(74.6)$ \\
\hline Abnormal oral diagnostic tests & $3664 / 4504(81.3)$ & $555 / 679(81.7)$ & $301 / 330(91.2)$ & $42 / 63(66.7)$ & $93 / 144(64.6)$ \\
\hline Unstimulated whole salivary flow & $2625 / 3546(74)$ & $430 / 571(75.3)$ & $253 / 292(86.6)$ & $35 / 58(60.3)$ & $93 / 144(64.6)$ \\
\hline Parotid sialography ${ }^{\pi}$ & $655 / 813(80.6)$ & $14 / 22(63.6)$ & 31/35 (88.6) & $1 / 2(50)$ & NP \\
\hline Salivary scintigraphy" & $1880 / 2262(83.1)$ & $179 / 194$ (92.3) & $74 / 89(83.1)$ & $12 / 14(85.7)$ & NP \\
\hline Positive anti-Ro/La antibodies & $4524 / 6154(73.5)$ & $896 / 1039(86.2)$ & $308 / 389(79.2)$ & $81 / 102(79.4)$ & $90 / 147(61.2)$ \\
\hline Anti-Ro antibodies & $4347 / 6152(70.7)$ & $873 / 1039(84)$ & 299/387 (77.3) & $79 / 101(78.2)$ & $80 / 147(54.4)$ \\
\hline Anti-La antibodies & $2669 / 6130(43.5)$ & $510 / 1027(49.7)$ & $180 / 390(46.2)$ & 40/102 (39.2) & $41 / 147$ (27.9) \\
\hline ANA positive & $4877 / 6062(80.5)$ & $876 / 1006(87.1)$ & 291/381 (76.4) & $87 / 104$ (83.7) & $117 / 145(80.7)$ \\
\hline RF positive & $2695 / 5549(48.6)$ & $488 / 954(51.2)$ & $202 / 384(52.6)$ & $49 / 97(50.5)$ & $39 / 145(26.9)$ \\
\hline C3 low & $684 / 5069$ (13.5) & $182 / 901(20.2)$ & $36 / 337$ (10.7) & $9 / 82(11)$ & $0 / 144(0)$ \\
\hline C4 low & $685 / 5064(13.5)$ & 91/901 (10.1) & $51 / 333(15.3)$ & $10 / 77(13)$ & $8 / 144(5.6)$ \\
\hline Positive cryoglobulins & $296 / 3664(8.1)$ & $3 / 266(1.1)$ & 4/128 (3.1) & $3 / 52(5.8)$ & $0 / 1(0)$ \\
\hline
\end{tabular}

*All comparisons were statistically significant (adjusted p-values for twenty comparisons with false discovery rate (FDR) correction < 0.05$)$ except for parotid sialography with a $p$-value equal to 0.086 .

NP: Not performed.

If P-value was computed excluding Others. 


\section{FIGURE LEGENDS}

Figure 1. Cluster bar charts for the \% of fulfilment of the 6 items of the 2002 classification criteria according to ethnicity.

Figure 2. Cluster bar charts for the \% of abnormal results in the immunological profile according to ethnicity.

Supplementary Figure S1. Missing data pattern.

Supplementary Figure S2. Cluster bar charts for the \% of fulfilment of the 6 items of the 2002 classification criteria of A) Asian patients diagnosed in non-Asian countries compared with B) native patients.

Supplementary Figure S3. Cluster bar charts for the \% of abnormal results in the immunological profile of A) Hispanic patients diagnosed in non-Latin American countries compared with B) native patients.

Supplementary Figure S4. Size of each cohort classified per city.

Supplementary Figure S5. Correlation between the percentage of biopsied patients and the percentage of Ro/La positive patients in each center. 